\title{
Contrôle de gestion et systèmes ERP : sens d'une relation
}

\author{
ERRAOUI Younes, and SLIMANI Hamid \\ ${ }^{1}$ Faculté des Sciences Juridiques Économiques et Sociales FES, MOROCCO \\ ${ }^{2}$ Faculté des Sciences Juridiques Économiques et Sociales FES, MOROCCO
}

\begin{abstract}
The emergence of ERP systems was significant in the mid-1990s, and most large entities adopted these global systems, which were presented as the "miracle solution" to multiple management problems. This paper first reviews the nature of these systems and the nature of management control. It then attempts to shed light on the relationship and expected impact of these information technologies on the performance of organizations. It is also suggested that the influence of ERP on the one hand makes the management control evolve more in its practical-functional aspects than in its conceptual dimension, and on the other hand it acts on cost analysis and on the decision-making process, but this has its requirements and conditions.
\end{abstract}

Keywords: Enterprise Resource Planning (ERP) - Management control - Information technology - Organizational performance.

Résumé : L'émergence des ERP s'est faite de manière significative au milieu des années 1990 et la plupart des grandes entités ont adopté pour ces systèmes globaux, présentés comme «la solution miracle » aux multiples problèmes de gestion. La présente communication fait d'abord le point sur la nature de ces systèmes et celle du contrôle de gestion. Elle tente ensuite de mettre la lumière sur la relation et l'impact prévu de ces technologies de l'information sur la performance des organisations. Il est aussi suggéré que l'influence des ERP d'une part, fait évoluer le contrôle de gestion plutôt dans ses aspects pratico-fonctionnels que dans sa dimension conceptuelle, et d'autre part elle agit sur l'analyse des coûts et sur le processus de prise de décision, mais cela a ses exigences et ses conditions.

Mots-clés : Progiciels de gestion intégrés (PGI) - Controle de gestion - Technologies de l'information - performance organisationnelle.

\section{INTRODUCTION}

Malgré l'importance accordée aux ERP par les chercheurs, de nombreuses questions continuent de se poser en raison de la complexité en termes de conception, de mise en œuvre, de propriété et d'évolution de cette technologie sophistiquée.

De nombreuses études indiquent que la mise en œuvre d'un système ERP peut être considérée comme un défi pour l'organisation en raison des multiples changements qu'elle peut apporter.

Une telle technologie " doit être capable de rassembler les gens et de les faire suivre des instructions bien écrites (...) elle doit séduire les gens pour qu'ils soient vigilants à son égard " (Latour, 2005). Le développement d'une technologie telle qu'un système ERP est considéré comme un processus d'inscription, qui peut être défini comme la construction d'un espace organisationnel sous forme de signes et de codes. 
Un certain nombre de chercheurs (Kanellou \& Spathis, 2013) ; (Wagner, Moll, \& Newell, 2011) rapportent que les caractéristiques spécifiques du système ERP contribuent au changement de la comptabilité de gestion. Ils rajoutent que l'intégration par la réingénierie des processus métier est un élément central de la mise en œuvre des systèmes ERP.

Aujourd'hui, l'émergence de notre question de recherche se situe au carrefour du système ERP et du contrôle de gestion, car d'une part, ce changement est lié à la nature même de la technologie et à son importance dans l'architecture technologique des organisations, et d'autre part, les systèmes ERP sont désormais la pierre angulaire de l'infrastructure managériale de toute organisation.

Ainsi, la mise en œuvre de ces systèmes est pilotée avant tout par la fonction de contrôle de gestion. Dans le processus d'intégration d'un système ERP, la plupart des entreprises commencent par le module "finance/contrôle de gestion". Ce module va définir l'architecture et la structure du système ERP pour l'ensemble de l'organisation.

Le défi lié à cette relation est important, car l'intégration des outils de gestion reste un problème majeur pour les organisations en termes de conception, d'utilisation et d'évolution. Le succès de ces outils de gestion est lié à la fois à la technologie elle-même et aux facteurs humains, ainsi qu'aux facteurs organisationnels.

La question de la relation entre le contrôle de gestion et les systèmes ERP est toujours au centre de l'intérêt des chercheurs et des praticiens pour de nombreuses raisons : les outils de gestion sont en constante évolution, de plus leur manipulation et leur utilisation nécessitent des spécialistes de plus en plus qualifiés et compétents. Leur intégration nécessite un plan de gestion du changement approprié qui prend en compte tous les facteurs de contingence comme moteur principal.

A cet égard, le succès du système ERP dans le contexte du contrôle de gestion semble pertinent pour plusieurs raisons. La première raison fait référence au rôle de la fonction de contrôle de gestion en termes d'analyse de l'information, de production d'indicateurs et de rapports de performance.

La deuxième raison renvoie au rôle de la fonction de contrôle de gestion en termes d'analyse stratégique et de reporting financier. La troisième raison renvoie à la place et à l'importance de certains outils technologiques de gestion tels que la Business Intelligence (BI), le Cloud Computing et les ERP dans la fonction de contrôle de gestion. La dernière raison fait référence à la divergence des résultats des études précédentes concernant l'impact de l'ERP sur la fonction de contrôle de gestion.

Le contrôle de gestion à son tour, est très souvent présenté dans les manuels comme un système de gestion générique, standard, adaptable à tous les types d'entreprises et à toutes les formes d'organisation.

A cet effet, le contrôle de gestion s'est largement ouvert à l'utilisation des ERP, et ce phénomène commence à faire émerger une panoplie d'interrogations et de nombreuses études, chose qui nous amène à s'interroger sur le sens de la relation existante entre les ERP et le contrôle de gestion.

Désormais et grâce à la technologie, le contrôle de gestion joue un rôle d'entonnoir dans la performance des organisations. De ce point de vue, nous pouvons souligner que le contrôle de gestion et l'ERP pourraient être considérés comme des sources de structuration de l'ordre social dans l'organisation. Cependant, la question qui se pose est la suivante : Quel est le sens et la nature de la relation entre le contrôle de gestion et les systèmes ERP ?

\section{Sous questions :}

$\rightarrow$ Comment l'intégration d'un système ERP influence-t-elle la fonction contrôle de gestion au sein des entreprises ?

$\rightarrow$ Le contrôle de gestion influence-t-il à son tours le recours à une solution ERP ? S'agit-il d'une relation unilatérale ou bien bilatérale?

$\rightarrow$ Comment l'interactionnisme structurationniste se crée entre l'ERP et le contrôle de gestion au sein de l'organisation? 


\section{CADRE CONCEPTUEL DE LA RECHERCHE : ESSAI DE SYNTHÈSE}

\section{a. Contrôle de gestion}

La première définition que donne R.N. Anthony du contrôle de gestion [(1965, p. 17) : «Le contrôle de gestion est le processus par lequel les responsables s'assurent que les ressources sont obtenues et utilisées avec efficacité et efficience dans l'accomplissement des objectifs de l'organisation. ». Cette définition s'élargit une vingtaine d'années plus tard pour devenir une véritable définition des modes de contrôle [ « Le contrôle de gestion est le processus par lequel les managers influencent d'autres membres de l'organisation pour appliquer les stratégies » (1993, p.35)].

En effet, outre le fait que dans cette deuxième définition l'accent est mis sur « les stratégies » plutôt que sur les « objectifs », elle introduit la notion d'influence des comportements des acteurs comme moyen de mettre en œuvre les stratégies de l'organisation.

L'idée est notamment reprise par Chiapello (1996, p.53) et par Langevin et Naro (2003, p.2) qui définissent le contrôle organisationnel comme «mécanisme dont la finalité est d'influencer les comportements des acteurs de l'organisation dans le sens attendu par cette dernière ».

Selon R N. Anthony toujours, « le CG est un processus par lequel les dirigeants influencent les membres de l'organisation pour mettre en œuvre les stratégies de manière efficace et efficiente». A. Burlaud et C. Simon considèrent que "le contrôle de gestion est un système de régulation des comportements de l'homme dans l'exercice de sa profession et, plus particulièrement, lorsque celle- ci s'exerce dans le cadre d'une organisation ».

Dans sa revue de littérature, E. Chiapello définit le contrôle en reprenant la notion d'influence rajoutée par Anthony en 1993 et en y incluant la notion d'intentionnalité suggérée par de Hopwood en 1974 : « Nous définissons le contrôle comme toute influence créatrice d'ordre, c'est-à-dire d'une certaine régularité. On est dans une situation de contrôle, selon cette définition, lorsque le comportement d'une personne est influencé par quelque chose ou quelqu'un. [...] Notre définition du contrôle englobe en fait les deux types de contrôle : celui mis au point par le management et celui qui émerge des interactions de toutes les influences pesant sur les comportements. [...] Pour faire le parallèle avec l'approche de Hopwood (1974), nous parlons de contrôle pour la résultante de l'ensemble des influences à l'œuvre, et des modes de contrôle pour définir les facteurs d'influence particuliers » (Chiapello, 1996, pp.53-54).

H. Mintzberg insiste sur l'importance de l'articulation entre les tâches et les acteurs qui doit accompagner la division du travail, et développe le concept de «mécanismes de coordination » qu'il décrit comme la « colle qui maintient ensemble les parties de l'organisation» (1982, p.19). Barel (2001, P.8) montre que les associations « division du travail coordination » de Mintzberg et « délégation d'autorité - contrôle » des théoriciens du contrôle relèvent des mêmes problématiques.

Le contrôle de gestion est présenté ici comme une fonction d'accompagnement du déploiement de la stratégie ; il permet de concrétiser les objectifs stratégiques au niveau de la gestion quotidienne et de formaliser les aptitudes ou le savoirfaire du quotidien au niveau stratégique.

Anthony offre également une vision managériale en soulignant l'implication des dirigeants dans le contrôle de gestion et dans la définition du modèle de performance. Il affirme enfin l'importance de la gestion du couple coût- valeur en reformulant le concept d'efficience. 
On peut dire que l'ensemble de ces auteurs soulignent le rôle du contrôle de gestion dans la coordination des comportements. Une organisation est constituée d'êtres humains poursuivant leurs objectifs propres qui sont généralement différents de ceux de l'organisation. Le contrôle de gestion met en place des dispositions qui conduisent à une convergence des intérêts individuels vers l'objectif de l'organisation chose qui nous mène à se baser sur la définition de Hopwood et H. Mintzberg qui définissent le contrôle de gestion comme un système de coordination créateur d'ordre et de régularité. Pour Simons, il définit le contrôle de gestion comme un système de frontières formel utilisé par le top management pour établir des règles qui doivent être respectées et des instructions à suivre. Ce système est conçu pour communiquer les risques qui pourraient se produire et qui doivent être évités afin que l'organisation puisse éviter toute action susceptible de déclencher l'apparition de risques indésirables ${ }^{1}$.

En général, on peut conclure que le contrôle de gestion est défini comme un système qui transmet des informations utiles aux managers pour une prise de décision efficiente et efficace afin d'atteindre les objectifs escomptés par l'organisation.

\section{b. Introduction aux Systèmes ERP :}

L'ERP est un système de gestion d'entreprise qui fournit une solution intégrée aux organisations pour répondre à leurs besoins en matière de traitement d'information, tout en gérant efficacement les ressources, c'est-à-dire les matières, les ressources humaines et financières, etc. ${ }^{2}$

Ainsi, on pourrait dire que l'ERP est un progiciel intégré de gestion, composé d'un ensemble de modules fonctionnels (logistique, finance, ressources humaines...) susceptibles d'être adaptés aux besoins de chaque client.

Un ERP combine la fonctionnalité de différents programmes de gestion en un seul, en se basant sur une seule base de données centralisée. On appelle souvent cette base de données le cube en informatique. Ceci permet de garantir l'intégrité et l'unicité des données auxquels les départements ont accès, en évitant de réintroduire les données, à chaque fois, de façon manuelle, dans les modules fonctionnels. Une facture qui aura été enregistrée dans le module commercial n'aura pas besoin d'être introduite à nouveau dans les modules comptables et financiers.

Selon Bansal (2015), l'ERP est un progiciel piloté par un système de gestion d'entreprise qui est intégré dans tous les aspects de l'entreprise. L'ERP est un progiciel complexe. Hwang et Min (2015) affirment que l'ERP peut générer des bénéfices de différentes manières, notamment en intégrant diverses activités dans un seul système, en facilitant le contrôle en termes de standards organisationnels, en améliorant l'accès à l'information en ligne et en temps réel, en améliorant les communications intra-organisationnelles et inter-organisationnelles, et en améliorant la capacité de prise de décision.

Ce type de système avec de telles caractéristiques peut être installé dans un environnement qui dépasse les frontières en supportant différentes langues, différentes devises et fiscalités.

D'une part, l'ERP peut ainsi générer facilement des documents conformes à la législation en vigueur pour chaque pays. Il s'agit d'un aspect important, à prendre en compte, vu la diversité des normes et des variations de celles-ci à travers le temps.

D'autre part, face à la demande du marché, des solutions spécifiques sont apparues pour les secteurs industriels. En effet, les fournisseurs des ERP ont développé des produits spécialisés qui incorporent des applications et des fonctionnalités.

\footnotetext{
${ }^{1}$ Ferreira A, Otley D. The design and use of performance management systems: an extended framework for analysis. Management Accounting Research 2009; 20(4): 263--282.

${ }^{2}$ Shih YY, Huang SS. The actual usage of ERP systems: an extended technology acceptance perspective. Journal of Research and Practice in Information Technology 2009; 41(3): 263--276.
} 
On peut dire qu'un ERP (enterprise requirement planning) est ce que l'on appelle plus communément un système intégré de gestion. Il permet à une entreprise de canaliser, sécuriser, et unifier les flux financiers des différents services qui la compose. Là où avant il y avait un système pour la gestion des achats, un autre pour la comptabilité, un troisième pour le contrôle de gestion, désormais, il n'y a plus qu'un seul progiciel.

\section{Figure 1 : Modèle classique de gestion des données}

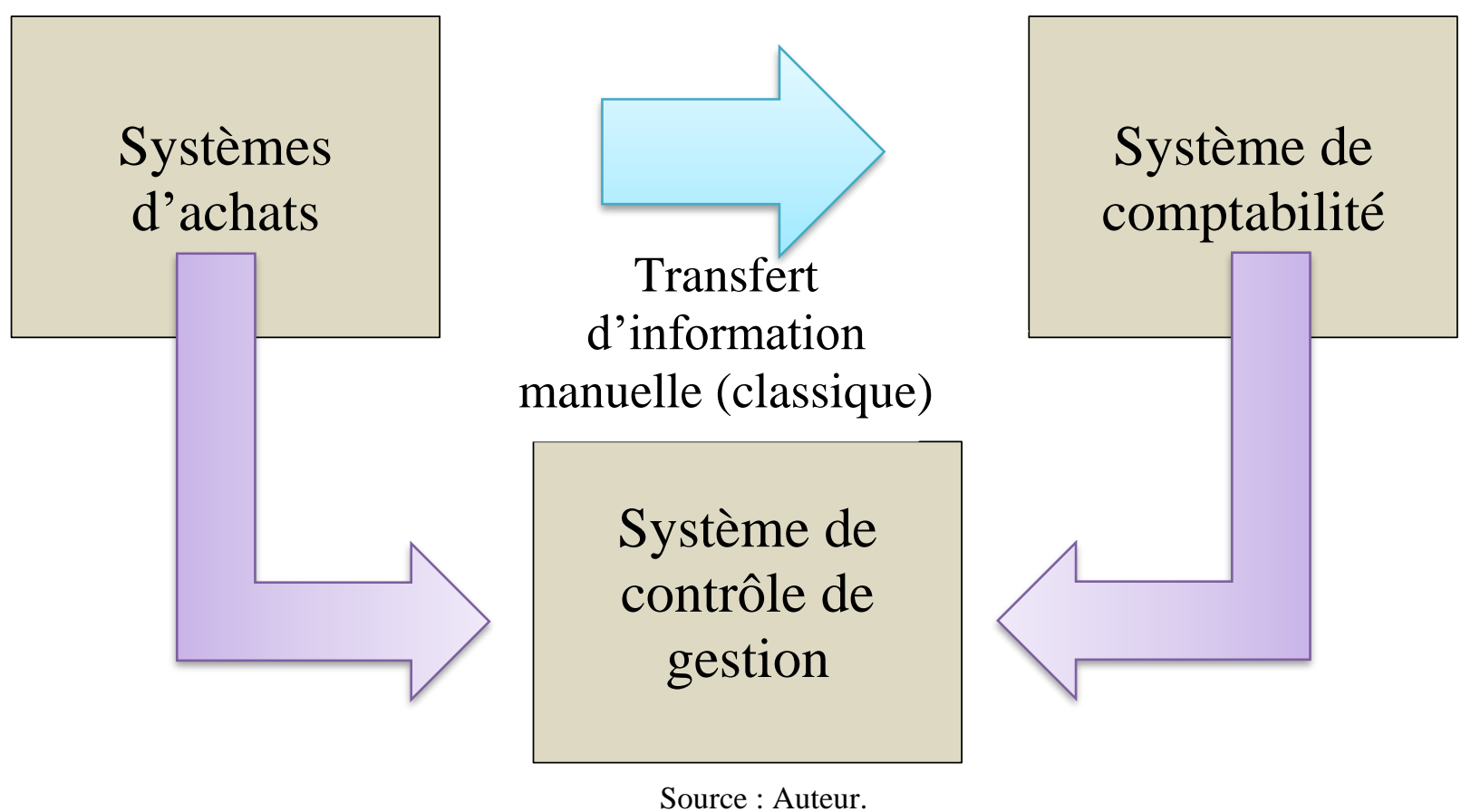

Dans l'exemple ci-dessus, on voit un mode de fonctionnement où l'entreprise utilise trois systèmes différents. L'information financière est donc à la charge de chacun de ses services, avec ses méthodes et conceptualisation du système.

Le risque d'avoir une information peu fiable est élevé. Chaque système transmet à l'autre par le biais d'une interface ou manuellement les informations. La signification d'un chiffre pour les achats peut être interprété différemment par la comptabilité. Enfin le fait de transmettre, vérifier et rassembler cette information est générateur de perte du temps.

A cet effet, l'ERP a la capacité d'une part de collecter et rassembler les flux qui se retrouvent au sein d'un même produit, et d'autre part il est capable de restituer une information sécurisée en temps réel, utilisable par tous, en fonction de leurs tâches spécifiques dans l'entreprise. 


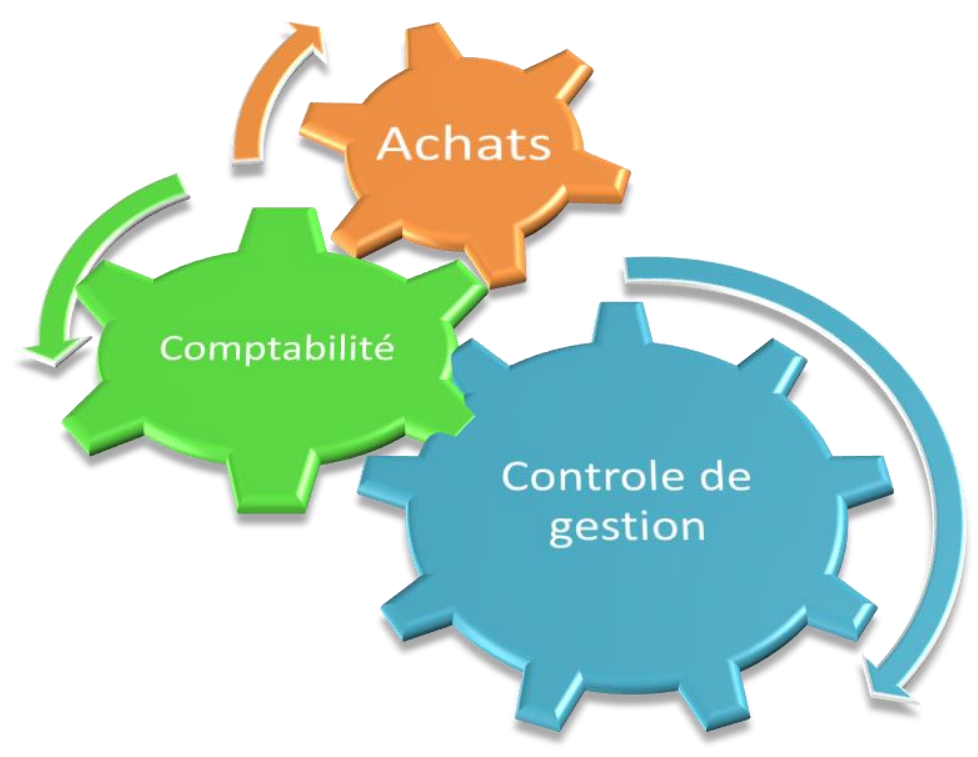

Source : Auteur.

Ainsi cette organisation centralisée des différentes fonctions au sein de l'entreprise procure une automatisation et une canalisation de traitement et de circulation des flux de données, aussi une unification des opérations répétitives, dégageant des gains de productivité spécifiques à ces différents domaines.

\section{LES FONDEMENT THÉORIQUES DE LA RELATION ERP/CG}

L'objectif assigné à ce paragraphe est d'établir d'un côté, les relations d'interdépendance entre la mise en place d'un système ERP et ses différents effets qui peuvent se nouer, en l'occurrence dans la fonction contrôle et d'autre coté, énoncer l'impact que peut jouer le contrôle de gestion sur le choix d'une solution ERP. Ces différentes interactions, nous allons les construire, à travers l'examen de plusieurs pans de la littérature théorique.

\section{a. L'ANT et la théorie de la structuration : quels apports ?}

- La théorie de l'acteur réseau :

Selon l'ANT, le système ERP peut être considéré comme un ensemble de pratiques de traduction et d'inscription qui rassemblent autour de lui un réseau d'acteurs humains et non-humains. Selon cette approche, l'ERP est vu comme une innovation qui sera appréhendée comme un construit socio-matériel dont le succès dépend de sa capacité à fédérer un réseau d'acteurs. La première étape de l'intégration du projet ERP consiste à définir et à répartir les rôles, les tâches et les responsabilités entre les acteurs internes et externes tels que les managers, les directeurs de département, les contrôleurs, les consultants et les autres membres du comité de pilotage. Basée sur le concept de sociologie de la traduction de (Callon, 1986), cette étape de l'intégration du projet ERP fait référence à la notion d'enrôlement qui signifie la manière de définir et de coordonner les rôles d'une part, et la manière de désigner un dispositif par lequel un ensemble de rôles interdépendants est défini et attribué aux acteurs qui les acceptent d'autre part (Callon, 1986). Ce concept d'enrôlement permet d'expliquer l'une des premières étapes de la phase de mise en œuvre du projet ERP. La deuxième étape de la phase 
de mise en œuvre de l'ERP dans la fonction de contrôle de gestion se concentre sur la définition des exigences des contrôleurs de gestion et de leurs besoins. Cela peut impliquer la redéfinition d'une nouvelle structure pour la fonction de contrôle de gestion par la possibilité d'introduire de nouvelles pratiques, processus, techniques et méthodes tels que $\mathrm{ABC}$, $\mathrm{ABM}$, etc. Au cours de cette étape, le comité de direction tente de définir un plan de projet pour répondre aux besoins d'information des parties prenantes. Ce plan vise à sensibiliser les futurs utilisateurs aux problèmes et aux avantages du système ERP. Nous pouvons analyser cette deuxième étape du projet ERP en nous référant aux concepts de "problématisation" et de "mobilisation". Le premier concept peut être considéré comme le résultat du processus par lequel les objectifs, les intérêts et les discours sont traduits en procédures et techniques de comptabilité et de calcul, (Robson, 1991). Le second concept fait référence à la mobilisation des ressources, à la construction de prototypes et à la proposition de démonstrations de la technologie en question. Cela permet de produire des arguments convaincants quant à la viabilité du système et sa légitimité afin de gagner le soutien des différents acteurs qui seront impliqués dans la réalisation du projet (Preston, Cooper, \& Coombs, 1992).

(Suchman, 2007) se réfère à la théorie de l'acteur-réseau pour construire sa réflexion sur la reconfiguration. Elle soutient que certaines propriétés peuvent être exclusivement humaines tandis que d'autres peuvent être matérielles. Elle insiste également sur la notion de "purification" de l'humain et du non-humain. Cependant, Latour, selon son concept de symétrie propose d'aller au-delà de cette notion, en suggérant " d'hybrider " ces deux identités que sont : l'humain et la machine. Dans le même sens, et selon l'ANT, l'interaction de ces deux entités dans un réseau peut être considérée comme le résultat de différents assemblages socio-matériels.

Selon la théorie de l'acteur réseau, les entités n'ont pas de qualités intrinsèques, mais elles acquièrent leurs formes et leurs attributs par l'interaction avec les autres dans la pratique. Dans la même optique, W. Orlikowski, (2009) affirme qu'il n'existe pas d'éléments sociaux ni d'éléments technologiques distincts et séparés. Ainsi, les éléments technologiques doivent être traités de manière symétrique aux humains.

\section{- La théorie de la structuration :}

Notre positionnement théorique converge ensuite envers une perspective structurationniste (Giddens, 1987 ; Orlikowski, 2000, 2011 ; De Vaujany, 2001, 2003, 2009). Comme le souligne De Vaujany, l'approche structurationniste des systèmes d'information est née au milieu des années 1980 : «Elle part d'une idée assez simple. Ce ne sont pas les outils informatiques qui sont innovants ou non, mais plutôt la manière dont les utilisateurs finaux se les approprient ».

Les structurationnistes s'intéressent à ces "innovations sociales d'usage", à leur forme et à leurs conditions d'émergence (De Vaujany, 2001).

Cette perspective s'appuie sur la théorie de la structuration de Giddens (1987). Ce corpus théorique propose d'appréhender un système social du point de vue de sa dynamique interne, dans un référent spatio-temporel, en articulant deux champs théoriques différents : le structuralisme et l'herméneutique.

D'une part le structuralisme, objectivisme où toute la réalité sociale est dans la structure globale qui s'impose aux acteurs, d'autre part l'herméneutique, subjectivisme où le sujet humain est la base essentielle dans la construction sociale, en dehors de la structure. Ainsi, l'idée de Giddens est d'appréhender tout système social comme un mouvement dans lequel les actions individuelles à la fois structurent les systèmes sociaux et sont structurées par eux. 
Un certain nombre de chercheurs ont mobilisé la théorie de la structuration de Giddens (1984) pour penser à la relation entre technologie et organisation. L'objectif principal de la mobilisation de la théorie de la structuration est d'évaluer son potentiel en tant que cadre théorique pour expliquer le succès de la technologie dans la fonction de contrôle de gestion. En considérant à la fois les aspects sociaux et matériels de la technologie, nous cherchons à comprendre le succès de l'intégration du système ERP dans la fonction de contrôle de gestion. La théorie de la structuration fournit des concepts théoriques permettant de comprendre le rôle du système de comptabilité et de contrôle dans la structuration de l'ordre social au sein de l'organisation. L'intégration d'une technologie dans l'organisation peut être un moyen qui renforce le contrôle sur les acteurs (domination chez Giddens), favorise la construction de sens chez les acteurs (signification chez Giddens) et enfin établit un certain aspect de gouvernance dans l'organisation en termes d'ordre moral (légitimation chez Giddens).

La technologie et le système de contrôle incorporent des propriétés structurelles et jouent un rôle dans la production et la reproduction du sens, du pouvoir et de la moralité dans l'organisation. Ils jouent également un rôle crucial dans la transformation et le changement des pratiques organisationnelles, des routines, des méthodes et des processus de gestion. L'intégration d'une nouvelle technologie est un déclencheur de changement au sein de l'organisation en termes de constitution de l'ordre social, des rôles, de la manière de faire, du système de contrôle et d'évaluation, mais aussi en termes de modification des propriétés institutionnelles. En nous concentrant sur l'intégration d'un nouvel outil de gestion dans la fonction de contrôle de gestion, nous étudions la question de la mise en œuvre réussie d'un outil de gestion par une meilleure intégration des codes, modèles, plans et règles. Pour une mise en œuvre réussie du système ERP au sein de la fonction de contrôle de gestion, ce système doit être capable d'intégrer les structures et les propriétés structurelles de la fonction concernée. La structure est considérée par (Macintosh \& Scapens, 1990, 1991) comme l'ADN de l'action sociale au sein de l'organisation, ils rapportent que les systèmes de comptabilité de gestion peuvent être considérés comme une modalité, soulignant que ladite comptabilité fournit aux gestionnaires des outils pour comprendre les activités de leur organisation en permettant une communication efficace entre activités. Ils considèrent le système de comptabilité de gestion comme un schéma interprétatif qui sert de médiateur entre la structure du sens et l'interaction sociale. Pour (Macintosh \& Scapens, 1990), les concepts comptables tels que les revenus, les actifs, les coûts et les bénéfices représentent la dimension du sens avant de devenir des schémas interprétatifs. En ce sens, les acteurs sociaux partagent d'abord la compréhension du sens à travers le système de comptabilité de gestion qui est également représenté par des schémas interprétatifs.

Au niveau de cette section nous avons abordé deux grandes théories qui peuvent nous aider à comprendre la relation entre l'intégration des ERP au sein des organisations et le contrôle de gestion.

En outre, ces deux théories présentes certaines différence. La principale différence entre la théorie de la structuration et l'ANT réside dans leur position sociotechnique.

Dans la théorie de la structuration, la capacité de toute technologie à faire la différence n'est pas reconnue. Elle est toujours reléguée aux outils ou aux installations techniques utilisés par les agents humains. Dans la théorie du réseau d'acteurs, la technologie devient un acteur indépendant à part entière, mais aucune distinction n'est faite entre le rôle de la technologie et celui des humains. Le système social et la technologie sont considérés comme inséparables.

Dans cette optique, un ensemble de chercheurs suggèrent que la théorie de la structuration exagère le rôle du facteur humaine dans la création et la production du contexte technologique et informationnel (Rose, Jones et Truex, 2005). 
Par contre, les partisans de l'ANT considèrent que ce contexte est à la fois social et matériel (ERP), c'est-à-dire un hybride d'acteurs humains et non humains.

Cependant, dans cette panoplie des acteurs, Walsham (2001) a apporté une contribution précieuse en combinant ces deux théories dans une seule optique, en utilisant la théorie de la structuration pour guider une analyse sociale plus large, et l'ANT pour décrire les processus sociotechniques détaillés qu'ont peu avoir suite à une intégration des SI.

\section{b. Attributions de l'approche socio-matérielle}

Un certain nombre de chercheurs adoptent une perspective socio-matérielle pour étudier comment les caractéristiques matérielles d'un système ERP s'entremêlent et évoluent conjointement avec le social dans la phase de post-implémentation (Wagner et al., 2010 ; Wagner, Moll, \& Newell, 2011). Cette perspective socio-matérielle considère que le matériel et le social sont mutuellement productifs. Pour eux, un système ERP est un assemblage socio-matériel habilitant et/ou contraignant de ce qui peut être accompli en termes de pratiques au sein de l'organisation. (Wagner et al., 2010) ajoutent que les désalignements entre les meilleures pratiques intégrées dans le système ERP et les pratiques de l'organisation sont hétérogènes. Pour parvenir à une synchronisation, une reconfiguration socio-matérielle est nécessaire. (Wagner et al., 2010) analysent les reconfigurations dans la phase de post-implémentation de l'ERP sur la base du processus de négociation entre les différentes parties prenantes et les différentes communautés de pratique afin d'atteindre un stade de stabilisation de la technologie.

L'essence de la perspective socio-matérielle réside dans le fait que le matériel et le social sont mutuellement constitués et, par conséquent, inséparables dans la pratique. En ce sens, les structures et les processus (par exemple, les règles et les routines associées à la configuration des meilleures pratiques) d'un système ERP sont en constante émergence.

(Wagner et al., 2010) décrivent que les routines et les règles liées à une meilleure pratique ne sont pas intégrées dans la technologie de l'information, mais émergent de l'utilisation du système dans la pratique. Ces pratiques émergent de l'interaction et de l'enchevêtrement du matériel et du social à travers le processus d'utilisation et de développement du système dans la phase de post-implémentation.

Par ailleurs, en s'appuyant sur une perspective socio-matérielle, (Wagner et al., 2011) rapportent que la comptabilité de gestion ne peut pas être facilement intégrée dans la structure de l'ERP, même si l'architecture technologique de ce système est censée être conçue pour s'adapter et s'intégrer facilement aux pratiques de l'organisation. Ils soulignent également le rôle et le pouvoir médiateur des communautés de pratique dans la mesure où ces logiques comptables familières peuvent être intégrées dans le système ERP. Ils ajoutent que la technologie ne peut à elle seule forcer le changement de pratique, surtout lorsque la logique des " meilleures pratiques " est mal alignée avec la logique des "anciennes pratiques" du contrôle de gestion. (Wagner et al., 2011) réalisent que l'architecture du système ERP n'est pas assez flexible pour permettre à certaines organisations de gérer les projets et les budgets comme elles le faisaient sous l'ancien système.

$\rightarrow$ L'approche socio-matérielle s'est beaucoup inspiré de l'ANT et des recherches de la théorie de la structuration. En effet, la sociomatérialité renvoie à un ensemble d'approches qui estiment que les outils et les acteurs sont consubstantiels dans une analyse des situations d'action (De Vaujany et Mitev 2015, Meissonier et Houzé 2018).

Les travaux de Callon et Latour (ANT) mettent en avant le caractère indissociable du social et du matériel, avec le principe de symétrie et la notion des actants au sein du même réseau (actants humains et non humains : technologies, ressources, objets...). Il ne s'agit donc plus d'étudier une simple interaction entre des acteurs d'un côté et des objets de l'autre, mais 
une véritable imbrication, des conjonctions et des disjonctions, jusqu'à une éventuelle émulsion qui peut prendre forme dans un réseau (qui devient à son tour un "actant" de l'innovation). A cet effet, les technologies (SI) ne sont plus de simples moyens d'exécution des actions humaines, mais elles participent d'une manière significative au réseau technicoéconomique, qui peut ou non, s'aligner et générer de l'innovation.

\section{METHODOLOGIE DE RECHERCHE}

Une méthodologie de recherche prend naissance à partir d'un problème à résoudre ou d'un mythe de compréhension et d'action. Ainsi, la conduite d'une recherche implique la mise en place d'un statut philosophique de recherche " épistémologique ", d'un objectif de recherche et d'un aspect technique.

Notre recherche s'inscrit dans une évolution des modèles d'organisation, qui a des répercussions sur les fonctions des entreprises et en particulier sur la fonction de contrôle de gestion et ses outils.

Nous pouvons dire que : " L'environnement des organisations est complexe et changeant, l'histoire est confuse, difficile à interpréter, l'expérience et les connaissances des acteurs des organisations ainsi que des étudiants sont limitées ".

Pour surmonter ces confusions, ces organisations créent et échangent des représentations de l'histoire qui leur permettent d'interpréter, de communiquer et plus généralement de vivre leur propre histoire. Ces récits sont socialement construits à partir d'un ensemble de mythes partagés ou de représentations conventionnelles.

Cet ensemble de connaissances repose implicitement sur l'idée que la réalité existe indépendamment des acteurs. Il offre la vision d'un monde bien mieux ordonné et contrôlé par les humains qu'il ne l'est réellement. " (March, 1981, p.37).

Ainsi, l'objectif de notre étude est d'établir les relations d'interdépendance entre la mise en place d'un système ERP et les différents effets organisationnels qui peuvent être établis, en l'occurrence dans la fonction de contrôle de gestion. Ces différentes interactions que nous allons découvrir, à travers l'examen de plusieurs parties de la littérature théorique et empirique, seront évaluées et testées empiriquement.

Compte tenu de la rareté des structures marocaines intégrant un système ERP comme mode de gestion organisationnelle, une approche qualitative est nécessaire pour mener notre étude de cas exploratoire, afin de lister les questions de recherche, les constats et les questions de recherche pouvant constituer la base de notre étude empirique (Yin, 1994).

Afin de structurer notre étude de recherche on a vu convenable de suivre :

- Épistémologie constructiviste ;

- Approche qualitative avec une démarche inductive ;

- Collecte de données via des interviews individuelles ;

- Des entretiens semi- directifs ;

- Méthode des cas.

Dans notre recherche nous avons utilisé une approche qualitative basée sur l'étude de cas multiples. Ce type de recherche va nous permettre d'examiner plusieurs cas afin de comprendre leurs similitudes et leurs différences (Baxter et Jack, 2008). L'objectif est de pourvoir comparer des entreprises disposant d'un ERP afin de comprendre les défis organisationnels et comportementaux auxquels leurs contrôleurs de gestion sont confrontés.

Merriam (2014), explique que l'inclusion de multiples cas représente une stratégie commune pour renforcer la validité externe. Elle permet d'avoir des interprétations plus convaincantes. Selon Miles et Huberman (1994), l'examen d'un nombre de cas contrastés permet de comprendre les résultats d'un seul cas et de pouvoir renforcer la précision, la validité et la stabilité des constatations dans les autres cas. 
Pour le choix des cas, nous avons utilisé une logique d'échantillonnage théorique. Les entreprises retenues dans notre recherche disposent d'un niveau d'intégration semblable de leur système ERP. Cependant, pour minimiser les facteurs de variabilité liés à la marque, nous avons choisi des entreprises qui disposent de la même marque d'éditeur : ERP SAP.

Selon Meyer (2001), ce type d'échantillonnage est fondamentalement différent de l'échantillonnage statistique. Son but est de sélectionner des cas qui sont susceptibles de reproduire ou d'étendre une théorie émergente, de remplir des catégories théoriques ou de fournir des exemples de type polaires (Eisenhardt, 1989).

\section{a. Première Étude de cas : Étude exploratoire}

L'objectif de notre étude est d'établir des relations d'interdépendance entre la mise en place d'un ERP et les différents effets organisationnels qui peuvent se nouer et influencer la fonction contrôle de gestion.

Ainsi à travers des entretiens semi-directifs auprès des personnes impliquées dans la mise en œuvre de ce système, nous avons pu étudier et observer les interactions de la relation existentiel entre les progiciels de gestion intégrés et le contrôle de gestion, tout au long du processus industriel de production de câblage au sein de la société Kromberg \& Schubert, chose qui nous a permis de déterminer et de cerner les facettes et l'ampleur de l'impact de cette relation, sur la performance de l'entreprise et surtout sur le processus du contrôle de gestion.

Dans la pratique, pour mener à bien ce travail, nous avons réalisé une enquête exploratoire sous forme d'entretiens individuels auprès d'un ensemble de personnes impliquées dans l'utilisation du progiciel et qui sont réparties sur les différents départements de l'entreprise. Ce premier entretien va nous permettre de structurer notre modèle de recherche afin d'adapter et affiner notre problématique de recherche.

Méthode de traitement

- Traitement des données qualitatives à partir du logiciel Nvivo 12 ;

- Entretien semi directif ;

- Questions ouvertes, où l'interviewé aura la liberté d'exprimé ses idées et son avis ;

- Transcription et interprétation des interviews.

\section{b. Guide d'entretien :}

Étant donné le vaste champ que nous souhaitons traiter avec cette étude exploratoire, on a conçu un entretien spécifique à l'ensemble des départements, et un entretien que pour le département de controlling et du IT, sachant que toutes les personnes interrogées avaient accès au système SAP. 
Tableau 1 : thématique de base du guide d'entretien

\begin{tabular}{|c|c|}
\hline Contenu & Objectif \\
\hline Thème I : Généralités & $\begin{array}{l}\text { Obtenir des informations sur l'expérience des } \\
\text { interviewés et tester leur connaissance à propos de leur } \\
\text { entreprise. }\end{array}$ \\
\hline Thème II : Fonction et acteurs du contrôle de gestion & $\begin{array}{l}\text { Mettre en exergue le rôle et l'implication du } \\
\text { département Controlling dans le suivi } \\
\text { d'accomplissement des taches au sein des différents } \\
\text { services de l'entreprise. }\end{array}$ \\
\hline Thème III : Introduction aux solutions ERP & $\begin{array}{l}\text { Explorer les connaissances et l'expérience des } \\
\text { interviewés vis à vis l'utilisation et le recours au } \\
\text { systèmes ERP afin de mesurer le degré d'implication } \\
\text { de ces systèmes dans l'exécution des différentes } \\
\text { taches. }\end{array}$ \\
\hline $\begin{array}{l}\text { Thème IV : Rôle des systèmes ERP dans l'intégration } \\
\text { de la fonction contrôle de gestion }\end{array}$ & $\begin{array}{l}\text { Découvrir dans quelle perceptive un système ERP } \\
\text { participe à l'accomplissement et l'exécution des } \\
\text { opérations au niveau du système hiérarchique et } \\
\text { surtout au niveau de département de contrôle de } \\
\text { gestion. }\end{array}$ \\
\hline $\begin{array}{l}\text { Thème V: Caractéristiques } \\
\text { ERP/CONTROLE DE GESTION }\end{array}$ & $\begin{array}{l}\text { Recueillir les spécificités de la relation entre les ERP } \\
\text { et le contrôle de gestion afin de dégager notre } \\
\text { perception d'instrumentalisation et d'intégration du } \\
\text { processus du contrôle de gestion. }\end{array}$ \\
\hline
\end{tabular}

\section{c. Caractéristiques de l'entreprise explorée et des profils des personnes interviewées}

Cette étude exploratoire donnera lieu dans nos futurs travaux, une confrontation plus vaste au terrain, s'il se vérifie que le sens de la relation entre les systèmes ERP et le contrôle de gestion est plus qu'une évolution pour les organisations.

Les personnes interviewées sont reparties sur l'ensemble du système hiérarchique de l'entreprise et ils sont tous impliquées dans l'utilisation du système ERP de type SAP S/4HANA.

L'interview s'est basée sur les grandes thématiques du questionnaire qui tient lieu de guide d'entretien. Étant donné le caractère exploratoire de ce travail, il s'agit de laisser parler la personne interviewée tout en la canalisant dans un axe principal de réflexion. 
Tableau 2 : Répartition des postes des interviewées

\begin{tabular}{|c|c|c|c|}
\hline Département & Nombre & Fonction & Durée \\
\hline Contrôle de gestion & 1 & CO manager & $30 \mathrm{~min}$ \\
\hline \multirow{2}{*}{ Finance } & 1 & FI manager & $20 \mathrm{~min}$ \\
\cline { 2 - 4 } & 2 & Comptables & $20 \mathrm{~min}$ \\
\hline $\begin{array}{c}\text { Technologie } \\
\text { d'information }\end{array}$ & 1 & IT manager & $20 \mathrm{~min}$ \\
\cline { 2 - 4 } & 2 & Techniciens IT & $20 \mathrm{~min}$ \\
\hline Logistique & 3 & Techniciens & $15 \mathrm{~min}$ \\
\hline Achat & 3 & Techniciens & $15 \mathrm{~min}$ \\
\hline Production & 2 & Techniciens & $15 \mathrm{~min}$ \\
\hline Total & 15 & 6 & $270 \mathrm{~min}$ \\
\hline
\end{tabular}

\section{d. Grille d'entretien semi directive}

Le but général de nos entretiens était de connaître le jugement de nos interlocuteurs sur le dispositif CG/ERP en général. A partir d'une grille de questions adaptée aux particularités des catégories des acteurs, nos entretiens visaient plus spécifiquement à connaître les opinions des acteurs du dite dispositif ERP, afin d'en ressortir son impact sur le Contrôle de gestion :

Axe I : Questions d'ordre général : présentation des interviewés

Au niveau de cette partie on a essayé de tisser les liens de contact avec les interviewés à travers une simple présentation tout en garantissant l'anonymat des intervenants.

Axe 2: Fonctionnement et acteurs du contrôle de gestion

Identifier les rôles et les liens de responsabilités résident entre le département « CONTROLLING », le top management (Niveau stratégique), le middle management et le First-line management (Niveau opérationnel).

Axe 3: Introduction aux technologies des systèmes ERP

Recueillir les connaissances et les expériences professionnelles des interviewés relatives à l'utilisation des systèmes ERP dans le but de dégager les facteurs influençant le recours aux systèmes ERP.

Axe 4 : Sens de la relation entre les systèmes ERP et la fonction contrôle de gestion

“'Les relations entre les services sont définies comme étant l'ensemble des interactions entre agents prestataires et agents bénéficiaires qui ne peuvent se résumer à de simples rencontres." E. Goffman.

Axe 5 : Caractéristiques de la relation ERP, Contrôle de gestion

Mettre la lumière sur l'impact lié aux interactions entre l'ERP, la fonction contrôle de gestion et les différents services et départements de l'entreprise.

\section{e. Analyse et discussion des résultats}

- Méthode de traitement

- Entretien semi directif;

- Questions ouvertes, où l'interviewé aura la liberté d'exprimé ses idées et son avis ;

- Traitement des données qualitatives à partir du logiciel Nvivo 12 ; 
- Transcription des interviews en se focalisant sur les énoncées permettant d'identifier les différents facteurs favorisant la production de l'information comptable à l'aide des systèmes ERP.

\section{- Transcription}

Toutes les entrevues individuelles réalisées avec les représentants du dispositif de contrôle de gestion ont été retranscrites, au moyen du logiciel QSR NVivo 12.

La finalité de cette recherche se trouve principalement dans le contenu des énonciations et non dans l'exhaustivité des opinions formulées. Analogiquement, on va intégrer des explications ou des références extratextuelles entre crochets pour faciliter la compréhension.

\section{- Codage des interviews}

La grille de codage sera développée sur le modèle thématique de l'entrevue en premier lieu, puis détaillée sur la base d'une lecture flottante. Un premier codage attribuait les principaux thèmes. La structure flexible du logiciel utilisé permet d'attribuer un même passage à plusieurs thèmes parallèlement, ce qui correspond à notre approche de questionnement où on a opté pour une structure ouverte sur plusieurs interviewés (postes occupés). Dans un deuxième et troisième pas, les grands thèmes vont être codifiés de manière plus détaillée, attribuant aux sections, par exemple, des valeurs pour des propositions affirmatives ou négatives dans le cas de questions de jugements ou structurant les thèmes principaux dans leurs sous-thèmes respectifs. Comme l'entretien semi-directif laisse une certaine latitude aux interviewés pour développer des sujets initialement non prévus dans la grille d'entretien, des thèmes supplémentaires vont être rajoutés au fur et mesure du codage.

\section{- Organisation de données}

L'analyse des entretiens est le résultat d'un choix thématique ainsi que d'un choix de citations. Le choix thématique était principalement guidé par nos questions de recherche les plus importantes. Il est évident, compte tenu de la structure ouverte de nos entretiens, que les experts interviewés vont aborder également des sujets en marge de nos questions centrales. Ainsi, si des représentants des IT, par exemple, vont évoquer des thèmes très spécifiques, liés principalement aux problèmes liés à leur postes ou leurs professions, nous allons les intégrées lors du codage. Ainsi ces thèmes vont uniquement être pris en considération quand un lien logique avec nos sujets principaux peut être établi. Le choix des citations, par contre, s'est orienté, d'une part, vers l'exemplarité d'une citation pour une certaine tendance d'opinion pointue par rapport à notre thématique.

\section{f. Les résultats :}

Afin d'analyser la relation existentielle entre le contrôle de gestion et les systèmes ERP, nous nous sommes basés tout d'abord sur l'observation et l'analyse des faits réels vécus lors de notre présence dans les locaux de l'entreprise Kromberg \& Schubert, puis nous avons réalisé une enquête exploratoire sous forme d'un ensemble d'entretiens individuels avec un groupement de personnes impliquées dans l'utilisation du progiciel et qui sont réparties au niveau des différents départements de l'entreprise.

Dans cette partie nous avons essayé de décomposer et de présenter les finalités du module de contrôle de gestion afin de mesurer plus précisément le sens et l'importance de l'utilisation d'un ERP dans l'accomplissement des fonctions de contrôle de gestion.

A cet effet, on peut dire que le système ERP peut apporter un ensemble de bénéfices, ainsi à travers le tableau suivant, nous avons essayé de présenter une liste non exhaustive des bénéfices observés suit à l'utilisation d'un système l'ERP. 
Tableau 3 : exemples des bénéfices observés du système ERP

\begin{tabular}{|l|l|}
\hline \multicolumn{1}{|c|}{ Fonctionnalité } & \multicolumn{1}{c|}{ Bénéfice } \\
Fixation du prix en temps réel sur les commandes des & Réduction des erreurs dans les prix. \\
\hline Identification automatisée des produits à livrer. & Réduction des erreurs et des retards des livraisons. \\
\hline $\begin{array}{l}\text { Possibilités de modification des opérations } \\
\text { d'expédition des commandes. }\end{array}$ & Respect des dates de livraison. \\
\hline $\begin{array}{l}\text { Suivi momentané des commandes des clients de la } \\
\text { cotation à la facturation. }\end{array}$ & Visibilité permanente sur l'état d'avancement des \\
\hline $\begin{array}{l}\text { Mise à jour automatisé des inventaires. } \\
\text { commandes. }\end{array}$ \\
\hline $\begin{array}{l}\text { Identification et sélection des coûts par produit ou par } \\
\text { projet selon la demande. }\end{array}$ & Une analyse des coûts plus claire et plus pointue. \\
\hline
\end{tabular}

Avec la multiplication actuelle et future de l'informatique au sein des entreprises, il est nécessaire que des outils soient créés pour harmoniser cette « jungle » d'information, surtout dans une période où l'information est un enjeu essentiel, que ce soit dans les groupes ou sur les marchés financiers. Au terme de notre étude, il nous semble que ce rôle peut être assumé et accompli par un système ERP.

Aujourd'hui, les systèmes ERP sont devenus l'une des préoccupations des centres de production automobile. En effet, leur mission consiste à rechercher une meilleure optimisation et synchronisation entre les différents flux et les vagues colossales d'informations.

On assiste ainsi, au développement des systèmes de contrôle formels visant à assurer une certaine réactivité stratégique face à un environnement économique turbulent, et à la pression concurrentielle en particulier. Le contrôle passe par une décentralisation des responsabilités de gestion vers les opérationnels.

Lors de notre stage chez Kromberg \& Schubert, nous avons étudié l'impact de cette décentralisation (normalisation) sur l'analyse des coûts et la prise de décision dans l'industrie de production de câbles automobiles. Après avoir analysé cet impact, nous avons proposé quelques pistes pour améliorer et optimiser le processus de contrôle et de maîtrise de ces coûts.

En effet, ladite décentralisation permet de percevoir plus rapidement et finement les évolutions du contexte nécessitant des ajustements stratégiques et une prise de décision momentanée. Afin d'assurer la coordination, la décentralisation implique également de développer un système de contrôle de gestion homogène et efficace. A cet égard, on peut dire que les systèmes ERP facilitent le mouvement de décentralisation par deux approches :

- ils assurent un certain contrôle des activités par la standardisation de l'information et la normalisation des référentiels d'action (Analyse et contrôle des couts);

- ils facilitent la communication entre les acteurs et la coordination des processus de contrôle stratégique, de gestion et opérationnel (Reporting et Prise de décision).

Ainsi, l'analyse des charges à travers l'ERP (SAP FICO) se fait selon deux modalités : l'analyse des frais généraux et l'analyse du coût par produit et/ou par projet. Ces deux éléments sont regroupés et régis au sein d'un périmètre analytique. 


\section{Analyse des frais généraux}

Le contrôle des frais généraux s'effectue par domaine de responsabilité et par éléments de coûts au sein de ce que SAP appelle des centres de coûts.

\section{$\checkmark$ Élément de couts "Cost element CE"}

La comptabilité financière et la comptabilité de gestion sont liées puisque chaque compte de résultat (FI) est relié à une nature comptable (CO). Par conséquent, chaque écriture dans la comptabilité financière crée des justificatifs de coûts sur les centres de coûts ou sur les ordres internes (objets de la comptabilité de gestion).

\section{$\checkmark \quad$ Les centres de coûts 'Cost centers CC'}

Les centres de coûts sont organisés au sein d'une hiérarchie analytique qui est univoque et obligatoire. Cette hiérarchie permet de fournir des données agrégées.

Un centre de coût représente une entité organisationnelle du plus bas niveau de l'entreprise.

\section{$\checkmark$ Ordres internes "Internal Orders"}

Les centres de coûts sont donc un service ou une subdivision d'un service. Ils restent très globaux et ne présentent pas un niveau de détail suffisant pour certains contrôles des coûts.

Afin de pallier ce manque de détail et d'avoir un outil de contrôle de gestion plus souple, on utilise une autre souscomposante du module $\mathrm{CO}:$ les ordres internes.

\section{$>$ Contrôle des coûts par produit}

Le contrôle des coûts par produit traite les différents aspects de la budgétisation et des coûts réels induits par la fabrication des produits et/ou services vendus.

Ce contrôle des coûts par produit se divise en trois catégories :

- Le calcul du coût de revient par produit ;

- Le calcul analytique des supports de coûts qui utilise un nouveau type d'ordre : les ordres de fabrication ;

- Le calcul du coût de revient réel.

\section{Contrôle des coûts par ordre}

A travers l'ERP, le contrôle des coûts peut s'effectuer de façon automatique grâce aux ordres. Ces derniers sont préétablis et chargés d'un budget en début d'année.

Lorsque l'on veut passer une commande, on peut paramétrer différentes réactions du système à la demande de dépenses en fonction d'un domaine de responsabilité (Ordres internes), d'un montant ou d'un pourcentage du budget.

Le domaine de responsabilité inclus un circuit de validation de la dépense. Les montants et les pourcentages sont eux les déclencheurs de circuit de validation.

Cependant, à travers cette présentation on peut confirmer que la contribution des ERP à l'efficacité du contrôle doit être comprise comme une ouverture à des choix organisationnels divers, qui peuvent être notamment soit de contrôle bureaucratique traditionnel, soit de double régulation.

Ainsi, l'ERP une fois intégré dans une démarche de changement organisationnel, il réfute les théories du contrôle bureaucratique traditionnel. Désormais, Il instaure un système de contrôle et de décision centralisé, avec une fonction contrôle de gestion cantonnée à un contrôle programmé (On parle désormais d'un Contrôle de gestion préventif). La décentralisation de responsabilités de gestion au niveau des opérationnels vise alors plus la diffusion d'un référentiel d'action normalisé (dans une logique de déploiement) qu'un objectif d'apprentissage organisationnel (dans le sens de l'émergence stratégique). 
L'ERP ne garantit pas le décloisonnement des processus managériaux. Il induit une formalisation des processus, qui peut correspondre à une stricte spécialisation fonctionnelle. Il offre ainsi une certaine flexibilité à l'utilisateur, dans le cadre de la possibilité de faire émerger de nouveaux indicateurs de performance. L'ERP peut également être mobilisé pour rationaliser les systèmes de gestion, sans exclure des outils plus souples et créatifs. En ce sens, l'organisation et le système de contrôle sont alors effectivement construits comme des systèmes de régulation doubles.

Pour conclure, il nous semble important de souligner l'enjeu qui définit le choix d'une solution ERP. Ce choix est fortement lié à la place et au rôle du contrôle de gestion dans le décloisonnement entre stratégie, organisation et action (facteurs de contingence). Dans le mouvement de développement du contrôle de gestion, la place et les missions assignées à cette fonction peuvent définir le choix d'un ERP. Il est fortement dépendant des représentations des acteurs telles que :

- le sens attribué au contrôle de gestion par la direction générale et les responsables opérationnels ;

- les compétences, l'histoire et la personnalité de l'organisation ;

- la légitimité de la fonction de contrôle de gestion à devenir une fonction omniprésente et polyvalente (capable d'intervenir à la fois sur les décisions opérationnelles et stratégiques).

Enfin, pour comprendre les configurations de contrôle et d'utilisation des ERP, la dimension processuelle de l'organisation est cruciale. C'est pourquoi il est important de suivre dans le temps le projet de mise en œuvre de l'ERP, l'évolution des systèmes de contrôle et le changement organisationnel. Au cours des différentes phases du changement (conception, changement et consolidation), les représentations des acteurs évoluent et définissent progressivement le sens de l'organisation et de sa performance.

Les implications de ces résultats en termes de gestion et de recherche seront testées et analysées dans un premier temps à travers l'enquête menée au sein de l'entreprise Kromberg \& Schubert, qui constitue notre première étude de cas, deux autres études de cas étant à venir.

\section{CONClusion :}

Le concept de contrôle de gestion a commencé à se développer. Un système de contrôle de gestion est défini comme un système qui transmet les informations les plus utiles aux gestionnaires dans les plus brefs délais afin d'assurer une prise de décision optimale dans le processus de gestion de la performance, qui est efficace et efficient pour la minimisation des coûts et la maximisation des profits, ce qui est nécessaire pour maintenir et améliorer l'avantage concurrentiel.

Par conséquent, les entreprises ont besoin de facteurs de soutien commercial, ce qui est censé être assuré par l'intégration d'un système ERP dans le processus de gestion des performances.

Ces ERP qui, à leur tour, sont censés aider à l'intégration d'un système de contrôle de gestion proactif capable d'aider la direction à prendre des décisions en temps opportun.

A cet effet, la tentative de combiner la théorie de la structuration et la théorie de l'acteur réseau ANT pour étudier le succès du système ERP dans la fonction de contrôle de gestion nous semble être la plus appropriée théoriquement, mais elle nécessite une certaine conscience des risques associés à la mobilisation de ces deux métathéories. Par ailleurs, cette combinaison offre une nouvelle perspective pour analyser de plus près les interactions entre le système ERP et la fonction de contrôle de gestion. Le cadre théorique combinant ces deux métathéories semble original et prometteur en termes de concepts que ces théories proposent et apportent à notre recherche. Inspiré par l'ANT et basé sur les recherches sur la théorie de la structuration, (Orlikowski, 2007, 2009) propose la nouvelle perspective socio-matérielle pour renouveler le 
corpus théorique de la recherche sur les systèmes d'information. Cette perspective postule que le matériel et le social sont constitutivement imbriqués dans la vie quotidienne (Orlikowski, 2009). Il semble que cette proposition trouve son fondement dans les travaux sur l'ANT et notamment dans le concept de symétrie qui considère les objets et les êtres humains comme des identités similaires appelées par B. Latour " actants ".

(Vernay et al., 2013) soulignent que l'ANT permet d'expliquer comment un réseau se constitue après l'intégration d'un système ERP via le processus de traduction. Ce dernier est considéré comme une clé pour comprendre la diffusion de l'innovation (Akrich et al., 1988 ; Callon, 1986).

Ainsi, notre modèle théorique, comme nous l'avons vu précédemment, se focalise sur la standardisation des processus qui est introduite avec l'introduction de l'outil ERP. Ce dernier renforce l'intégration organisationnelle par les mécanismes de coordination qu'il impose, et il a également un impact sur les mécanismes de contrôle formel en proposant de nombreux états de contrôle comme standard. D'autre part, cette standardisation est à son tour impactée par l'intégration qu'elle génère au sein de l'organisation, puisque cette dernière contribue à l'élaboration des normes les plus appropriées. De même, les mécanismes de coordination informels alimentent l'élaboration des normes. Par ailleurs, l'intégration créée par une telle normalisation des processus peut avoir un impact étroit sur les mécanismes de coordination du contrôle de gestion (formel et informel) tout en agissant sur les différents processus de l'organisation, dont la logique est devenue horizontale et transversale.

\section{Bibliographies:}

[1] Anthony, R. (1965). Management Planning and Control System: A Framework for Analysis, Harvard Business School Press ;

[2] Bansal V, Agarwal A. Enterprise resource planning: identifying relationships among critical success factors. Business Process Management Journal 2015; 21(6): 1337 - 1352 ;

[3] Bouquin, H. (2006). Le contrôle de gestion. Dunod. 7e edition ;

[4] Bouquin H. (2001), Le contrôle de gestion, PUF, Paris (5e éd.) ;

[5] Bouquin (1994), Les fondements du contrôle de gestion. Presse universitaire de France «Que sais-je ? ; №2892 ; Paris

[6] Hopwood, A.G., 1987. The archeology of accounting systems. Accounting, Organizations and Society 12, 207-234 ;

[7] Hwang D, Min H. Identifying the drivers of enterprise resource planning and assessing its impacts on supply chain performances. Industrial Management \& Data Systems 2015 ;

[8] Lorino P. (1997), Méthodes et pratiques de la performance, éd d'organisation, Paris ;

[9] Macintosh, N., \& Quattrone, P., 2010. Management Accounting and Control Systems: An Organizational and Sociological Approach. John Wiley \& Sons Ltd ;

[10] Orlikowski, W.J., 2005. Material Knowing: The Scaffolding of Human Knowledgeability. Sixth European Conference on Organizational Knowledge, Learning, and Capabilities. Cambridge, U.S.A ;

[11] Orlikowski, W.J., 2010. The Sociomateriality of Organisational Life: Considering Technology in Management Research. Cambridge Journal of Economics 34(1), 125-141 ;

[12] Philippe Lorino (2003), "Méthodes et pratiques de la performance - Le guide du pilotage ", Editions d'Organisation ;

[13] Simons R. How new top managers use control systems as levers of strategic renewal. Strategic Management Journal 1994 ;

[14] Simons R. (1995), Levers of control, Harvard Business School Press, Boston ;

[15] Wagner, E., Newell, S., \& Piccoli, G., 2010. Understanding project survival in an ES Environment: A sociomaterial practice perspective, Journal of the AIS 11(5), http://aisel.aisnet.org/jais/vol11/iss5/1/;

[16] Wagner, E., Newell, S., 2006. Repairing ERP: Producing Social Order to Create a Working Information System. Journal of Applied Behavioral Research 42(1), 40-57. 